\title{
Experimental implementation and proof of principle for a radionuclidic purity test solely based on half-life measurement
}

\author{
Jørgensen, Thomas; Jensen, Mikael
}

\section{Published in:}

Applied Radiation and Isotopes

Link to article, DOI:

10.1016/j.apradiso.2015.12.038

Publication date:

2016

Document Version

Peer reviewed version

Link back to DTU Orbit

Citation (APA):

Jørgensen, T., \& Jensen, M. (2016). Experimental implementation and proof of principle for a radionuclidic purity test solely based on half-life measurement. Applied Radiation and Isotopes, 108, 108-115.

https://doi.org/10.1016/j.apradiso.2015.12.038

\section{General rights}

Copyright and moral rights for the publications made accessible in the public portal are retained by the authors and/or other copyright owners and it is a condition of accessing publications that users recognise and abide by the legal requirements associated with these rights.

- Users may download and print one copy of any publication from the public portal for the purpose of private study or research.

- You may not further distribute the material or use it for any profit-making activity or commercial gain

- You may freely distribute the URL identifying the publication in the public portal 


\title{
Experimental implementation and proof of principle for a Radionuclidic Purity test solely based on half life measurement
}

\author{
Thomas Jørgensen ${ }^{\mathrm{a}, *}$, Mikael Jensen ${ }^{\mathrm{a}}$ \\ ${ }^{a}$ Hevesy Lab, DTU-Nutech,Technical University of Denmark, DK-4000 Roskilde, \\ Denmark
}

\begin{abstract}
In this paper we present the results of an experimental implementation of the method [1] for testing the radionuclidic purity (RNP) of F-18 compounds.

The overall limitations of the experimental methods and their possible impacts on RNP detectability have been identified. We have developed an GUI application for use as an easy and automated test tool in the production procedure.

The test results show that this method fully complies with the requirements in the European Pharmacopoeia (Eur. Ph.).

Keywords: Radionuclidic Purity, F-18, Software, Statistical test, half life, deadtime correction
\end{abstract}

\section{Introduction}

The widespread use of short lived radiopharmaceuticals for PET have demonstrated the complexity of establishing radionuclidic purity (RNP) spanning a product shelf life of several half-lives. A very important example is F-18 compounds with half-life about 2 hours. Here a shelf life of 10 hours with a RNP specification $>99.9 \%$ (as current European Pharmacopoeia requires) necessitates the detection of a long lived impurity containing 1/32000 of the activity at time of release.

We have in a previous paper [1] examined the mathematical background for using pure half-life determinations to establish such purity levels.

*email: sam_malone@me.com 
Now we have tested several practical implementations of the half-life method. In this paper we describe the overall limitations of the experimental methods and their possible impact on RNP detectability. The important factors of detector dynamic response and linearity range, signal-to-background, dead time and timing accuracy have been identified in the experimental implementation and their impacts on method sensitivity found.

\section{Theory}

The RNP test is based on a two component decay model of F-18 and one other contaminating isotope with a half-life of $\beta T_{R}$, where $T_{R}$ is the half life of F-18. The level of contamination at the time $t=0$ is $\alpha$, where $\alpha$ is defined as the fraction between the count rates of the contaminating isotope and F-18, $\alpha=\frac{A_{\text {other }}}{A_{F-18}}$.

The number of counts $N(t)$ is described over time by the equation:

$$
N(t)=\frac{N_{0}}{1+\alpha}\left(\left(\frac{1}{2}\right)^{t / T_{R}}+\alpha\left(\frac{1}{2}\right)^{t /\left(\beta T_{R}\right)}\right)
$$

where $N_{0}$ is the initial number of counts.

The RNP is defined as:

$$
R N P=\frac{1}{1+\alpha}
$$

\subsection{Determination of $N_{0}$ and the associated uncertainty}

The RNP test is based on a comparison of theoretical and measured data for a decay and therefore the initial number of counts $N_{0}$ in the exponential decay is of great importance. Thus we have to determine this as accurate as possible from the measured data to establish the theoretical basis for comparison. The limits for RNP, $\beta$ and time of measurement have to be corrected in order to adapt the associated uncertainty of $N_{0}$.

In [1] we proposed that $N_{0}$ is found as an average of the first 10 data points (normalized), but this gives rise to a systematic error, since a data set with an impurity will cause a value of $N_{0}$ that deviates from the "true" value, and this will affect the test result.

For impurities with $\beta<1$ the estimated value of $N_{0}$ will be smaller than the true value and vice versa. 
Instead we determine $N_{0}$ from an exponential fit of the data points within $t \ll t_{c}, t_{c}$ being the time (in minutes) where the impurity starts to dominate, given by:

$$
t_{c}=\frac{\ln (1 / \alpha)}{\ln (2)} \frac{\beta}{\beta-1} T_{R}
$$

Figure 1 shows a graph of $t_{c}$ (in minutes) as a function of $\alpha$ and $\beta$ and it is seen, that we safely can make a fit of the data points within the first hour or two for all relevant isotopes and degrees of impurities.

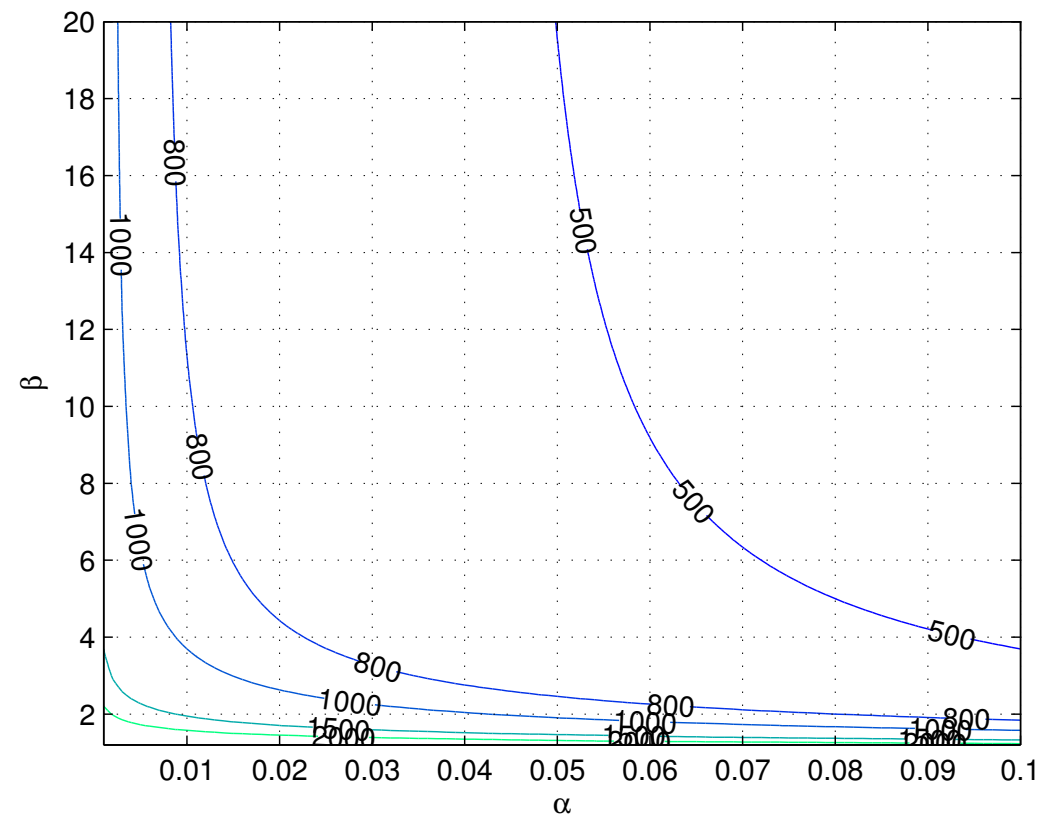

Figure 1: The figure shows a graph of $t_{c}$ (in minutes) as a function of $\alpha$ and $\beta$.

In this interval the deviation between the pure and impure curve will be very small. The relative deviation can be found as:

$$
d(\%)=\frac{N(t)_{\text {impure }}-N(t)_{\text {pure }}}{N(t)_{\text {pure }}} \cdot 100 \%=\frac{\alpha}{1+\alpha}\left(\left(\frac{1}{2}\right)^{\frac{1-\beta}{\beta T_{R}} t}-1\right) \cdot 100 \%
$$

Figure 2 shows the relative deviation (in \%) between the pure and impure graph after the first hour, and it is clear that deviations that small will have no significant impact on the determination of $N_{0}$. 


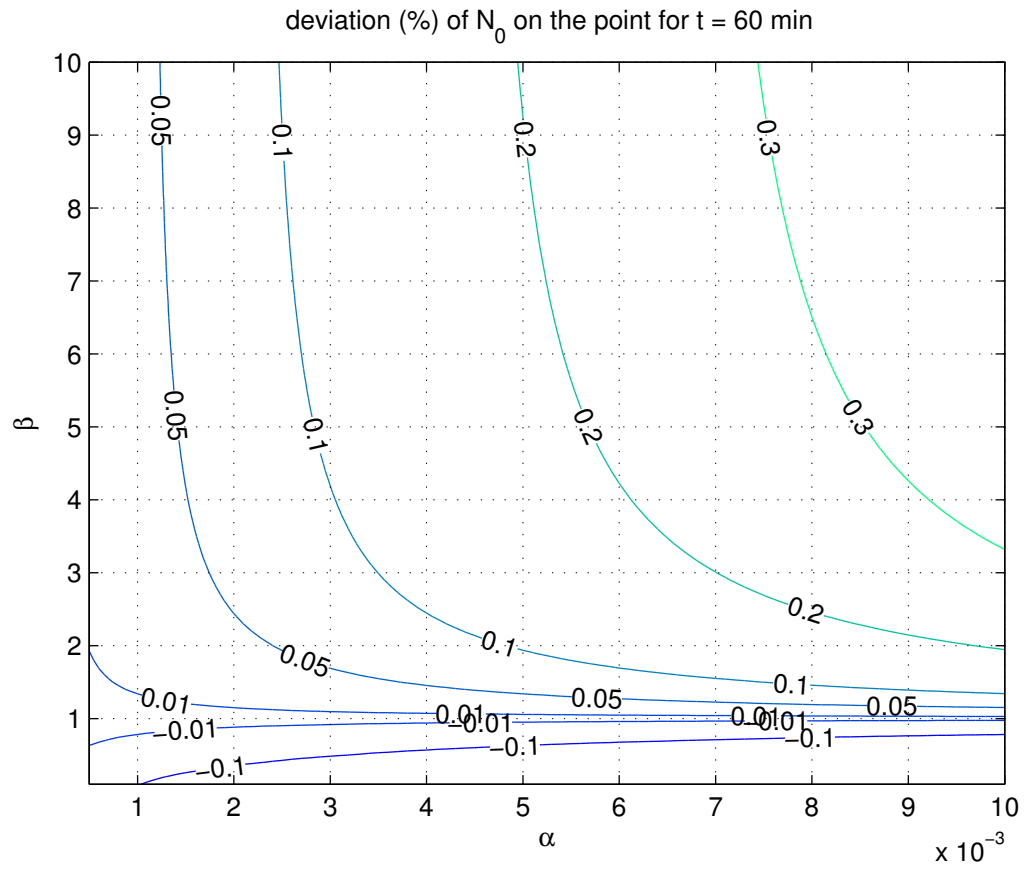

Figure 2: The graph shows the relative deviation (in \%) between the pure and impure graph after the first hour as a function of $\alpha$ and $\beta$. 
The uncertainty $\sigma_{N_{0}}$ of $N_{0}$ is the standard deviation found from the calculation of the exponential fit. In this calculation we have included the poisson statistical uncertainty on the first few measurements contributing to $N_{0}$.

\subsection{Uncertainty of time}

Timing imperfections in the detector chain used increase the uncertainty of each measurement. One example of that, could be the uncertainty in time, which can be converted to an equivalent uncertainty in the count value by:

$$
\sigma_{\text {equiv,time }}=\left|\frac{d N(t)}{d t}\right| \cdot \sigma_{\text {time }}
$$

From this (see appendix A) it is obvious that the uncertainty in time will have a greater influence on the counts, the higher the count rate is. The

question is then, how large the initial counts can be without the equivalent uncertainty gets dominating. We choose $\sigma_{\text {equiv }} \leq \sigma_{\text {poisson }}$ :

$$
\frac{\log (2)}{T_{R}} \cdot N_{0} \cdot\left(\frac{1}{2}\right)^{t / T_{R}} \cdot \sigma_{\text {time }} \leq \sqrt{N_{0} \cdot\left(\frac{1}{2}\right)^{t / T_{R}}}
$$

which gives (for $t=0$ ):

$$
N_{0} \leq\left(\frac{T_{R}}{\log (2) \cdot \sigma_{\text {time }}}\right)^{2}
$$

Using the timing error in the counter setup as the uncertainty in time, $\sigma_{\text {time }}=1 / 14$ min (see results section), we get the value of the initial counts, $N_{0} \leq 5.1 \cdot 10^{6}$. Below this limit the poisson noise forms the greater part of the uncertainty, see figure 3 .

\subsection{Total uncertainty of the data points}

The uncertainty of $N_{0}$ propagates through the calculations and contributes to the uncertainty of each data point. This is calculated in appendix B.

Hence, the total uncertainty for each data point in the "theoretical decay" is found by:

$$
\sigma_{\text {total }}=\sqrt{\sigma_{\text {equiv,time }}^{2}+\sigma_{N(t)}^{2}+\sigma_{\text {poisson }}^{2}}
$$




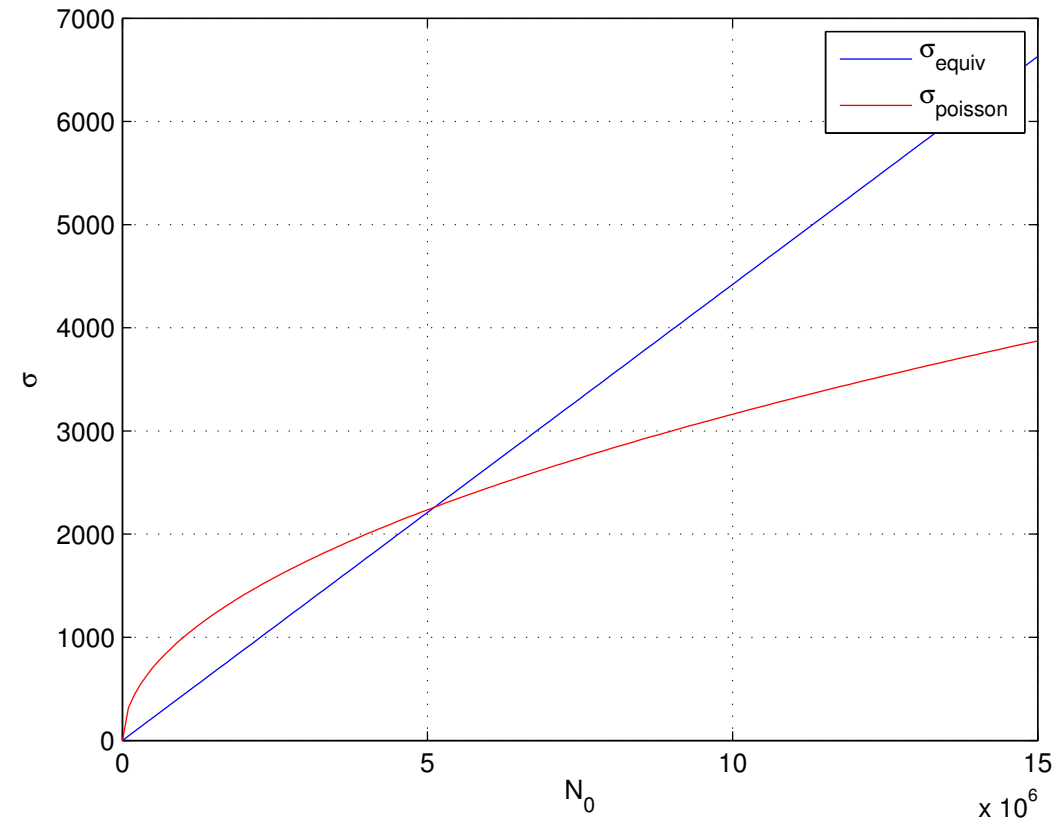

Figure 3: The figure shows a comparison of the equivalent and the poisson uncertainty. The value of the initial counts should be less than $5.1 \cdot 10^{6}$ (for $\sigma_{\text {time }}=1 / 14 \mathrm{~min}$ ) in order to minimize the influence of the uncertainty in time relative to the poisson noise. 


\subsection{Normalized uncertainty as limits in the test}

In the test the decay measurements are normalized by the factor $c=$ $\left(\frac{1}{2}\right)^{-t / T_{R}}$, where the uncertainty of time again contributes to the total uncertainty of the normalized (theoretical) data points. The total relative uncertainty of the normalized data points is (see appendix C):

$$
\frac{\delta c}{c}=\frac{\log \left(\frac{1}{2}\right)}{T_{R}} \cdot \sigma_{\text {time }}=\lambda \cdot \sigma_{\text {time }}
$$

where $\lambda$ is the decay constant. Hence, it is possible to find $\sigma_{\text {norm }}$ for use as the limits in the test. In figure 4 the normalized total uncertainty $\sigma_{\text {norm }}$ is compared to the normalized poisson noise, and it is seen that $\sigma_{\text {norm }}$ is larger in the first part of the measurement, while they approach each other in the last part. The graph is based on an example with $N_{0}=5000000, \Delta t=5$ $\min , \sigma_{\text {time }}=0.07 \mathrm{~min}$ and $\sigma_{N_{0}}=1000$.

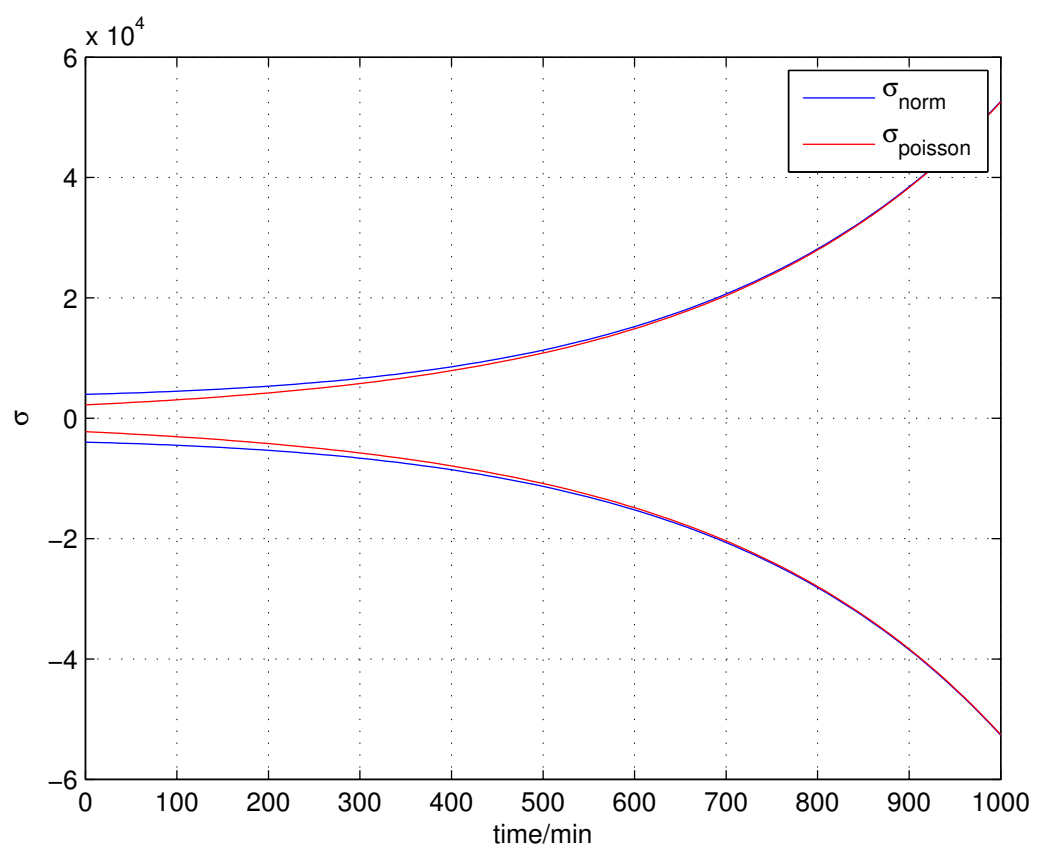

Figure 4: The figure shows a comparison of the poisson and the total uncertainty - both normalized. An example with $N_{0}=5000000, \Delta t=5 \mathrm{~min}, \sigma_{\text {time }}=0.07 \mathrm{~min}$ and $\sigma_{N_{0}}=$ 1000 . 
In the RNP test we calculate the averages of the normalized counts up to each point of time and find the associated uncertainties [1]. Figure 5 shows the uncertainties of the averages to each point of time for an example with the same values used above.

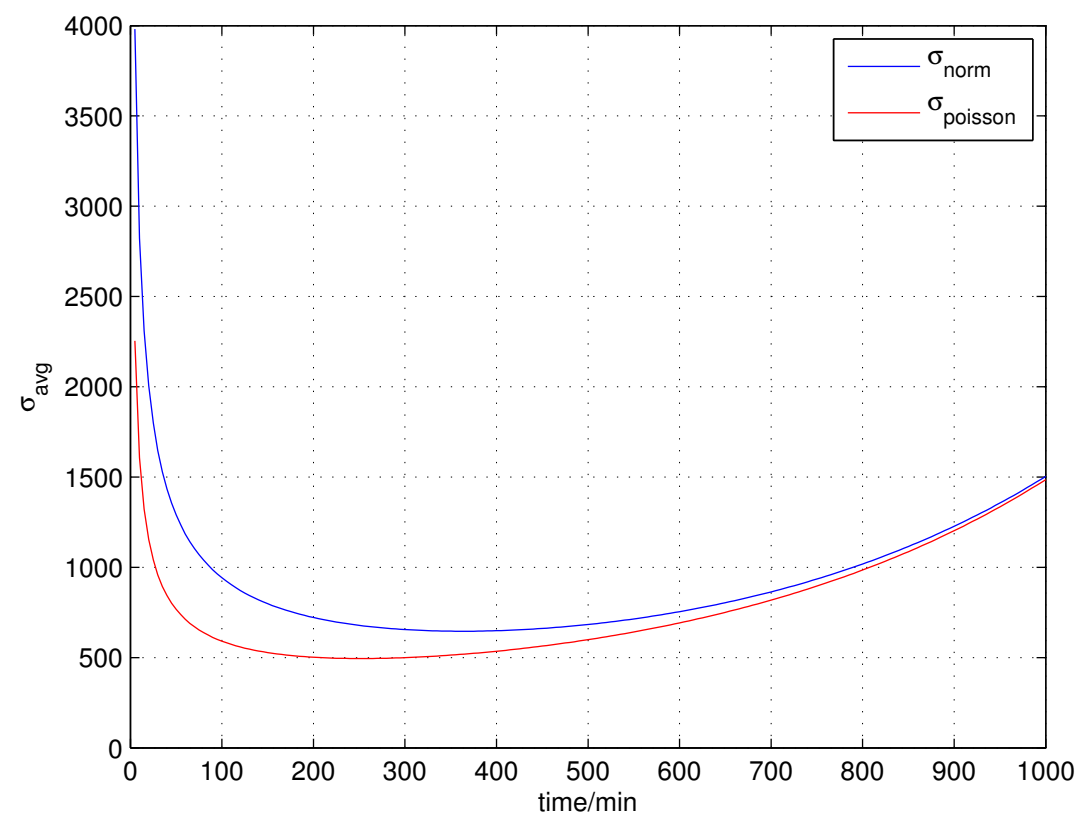

Figure 5: A comparison of the poisson and total uncertainty of the averages to each point of time for a pure sample with $N_{0}=5000000, \Delta t=5 \mathrm{~min}, \sigma_{\text {time }}=0.07 \mathrm{~min}$ and $\sigma_{N_{0}}=$ 1000 .

On the basis of $N_{0}$ and the calculated uncertainties it is now possible to say how many data points from the data set that are needed to test for the chosen test parametres $(R N P, \beta)$. This is done by finding the interception between the limits for the pure and impure $(R N P, \beta)$ sample of $\mathrm{F}-18$, see figure 6 . Then the test is carried out by calculating the average of the normalized data points up to this time limit (interception) and see whether the value lies in or out of the pure limit. 


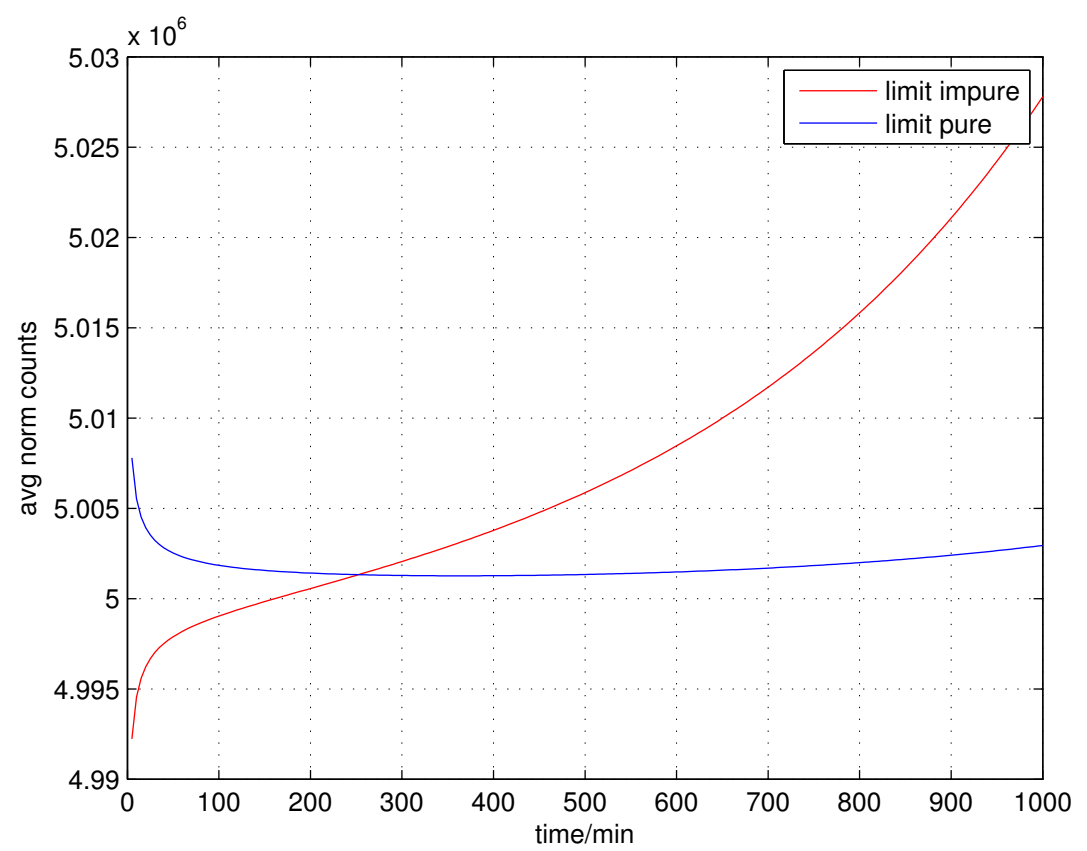

Figure 6: The figure shows an example of the limits for a pure $\left(+1.96 \sigma_{\text {norm }}\right)$ and an impure $\left(-1.96 \sigma_{\text {norm }}\right)$ sample of F-18 with $N_{0}=5000000, R N P=99.9 \%, \beta=2, \Delta t=5$ min, $\sigma_{\text {time }}=0.07 \mathrm{~min}$ and $\sigma_{N_{0}}=1000$. The interception of the two limits (here $255 \mathrm{~min}$ ) gives the necessary number of data points for the test. If the average of the measured data points up to this time lies within the value of the blue limit at this point, the sample is pure. Otherwise it is impure. 


\section{Material and methods}

We have collected data with two different kinds of counter setups. One, with a scintillation counter containing a NaI crystal (COBRA, Canberra Packard GMbH Schwandorf, Austria), and two, with a liquid scintillation counter LSC (LKB Wallac 1214 Rackbeta Counter, LKB Instruments, Australia). In the COBRA counter setup the counter corrects for dead time automatically, whereas data from the LSC require a manual correction.

The COBRA counter is a $\gamma$-only NaI scintillation detector and is "blind" to isotopes only emitting $\beta$ radiation.

The data were obtained by measuring the decay of a number of FDG samples, that were assumed to be pure and also on samples that were contaminated manually. The counts were measured within full energy spectrum, since the test is based on a comparison of total activities of two isotopes according to the definition of RNP. The measurements were done with counting periods of both $300 \mathrm{sec}$ and $600 \mathrm{sec}$ in order to find the best procedure balancing the choice of having enough data points in the beginning and enough time in total to see small contaminations. This is due to the fact that the LSC limits the number of measurements to 99 without removing the sample from the system.

The activities of the samples were in the order of $1 \mathrm{e} 4-1 \mathrm{e} 5 \mathrm{cps}$, and they were contaminated in a degree of $\alpha=0.05-0.1 \%$, see table 1 .

The dead time and the associated uncertainty in the LSC has been determined by measurements on 10 data sets with the same activities and 10 with varying activities using the decaying source method with the non-paralysable model (fixed dead time) from [2].

The background measurements were carried out with samples (LSC vial with cocktail but no added activity) counted after the main sample using exactly the same data collection protocol, and the average of these measurements is used as the background value in the test. If the measurements are isolated from external influences, the variation in the back ground should only be poisson noise.

We have developed an GUI application written in MATLAB (Mathworks, Kista, Sweden) that is based on the second method - the 'trumpet-mean model' - presented in [1], which was used to test all the data.

The fundamental algorithm is unchanged, but important improvements have been made to add dead time correction, back ground subtraction and a method for detecting and correcting timing "glitches", when the detector 


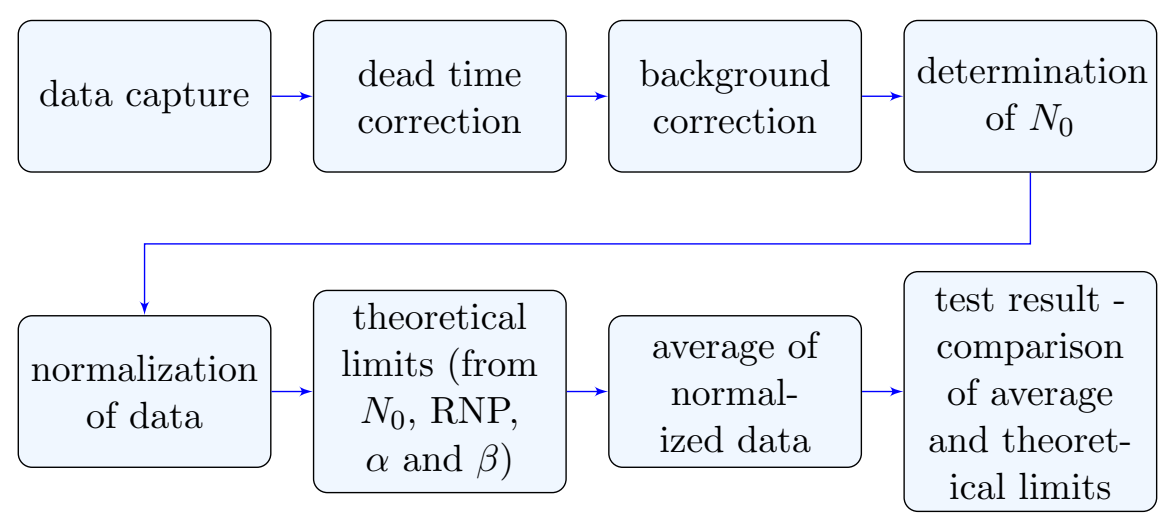

Figure 7: The figure shows a flow diagram over the RNP test procedure.

system only reports a time stamp in whole numbers of minutes.

\begin{tabular}{|c|c|c|c|c|}
\hline isotope & Ti-45 & Cu-64 & Mo-93m & H-3 \\
\hline half-life & $184.8 \mathrm{~m}$ & $12.70 \mathrm{~h}$ & $6.85 \mathrm{~h}$ & $12.32 \mathrm{y}$ \\
\hline$\beta$ & 1.68 & 6.94 & 3.74 & 58991 \\
\hline$\alpha$ & $0.1 \%$ & $0.05 \%, 0.1 \%$ & $0.05 \%, 0.1 \%$ & $0.05 \%, 0.1 \%$ \\
\hline method & sim data, data & sim data, data & sim data, data & sim data \\
\hline result & impure & impure & impure & impure \\
\hline
\end{tabular}

Table 1: Tested isotopes with contamination levels of $\alpha$. All impurities were found in the test by adjusting the RNP test level.

\section{Results}

We have tested several samples of FDG in order to see, how the RNP-test works with real data. Based on these measurements we emphasize a number of things that have decisive influence on the test result.

The final GUI is shown in figure 8. The user must select which impurity ( $\beta$ value) to look for and to what level of purity (RNP value) the statistical test should be performed.

The application allows RNP determination for other isotopes, as long as the principal half-life is entered correctly. However, all the above considera- 


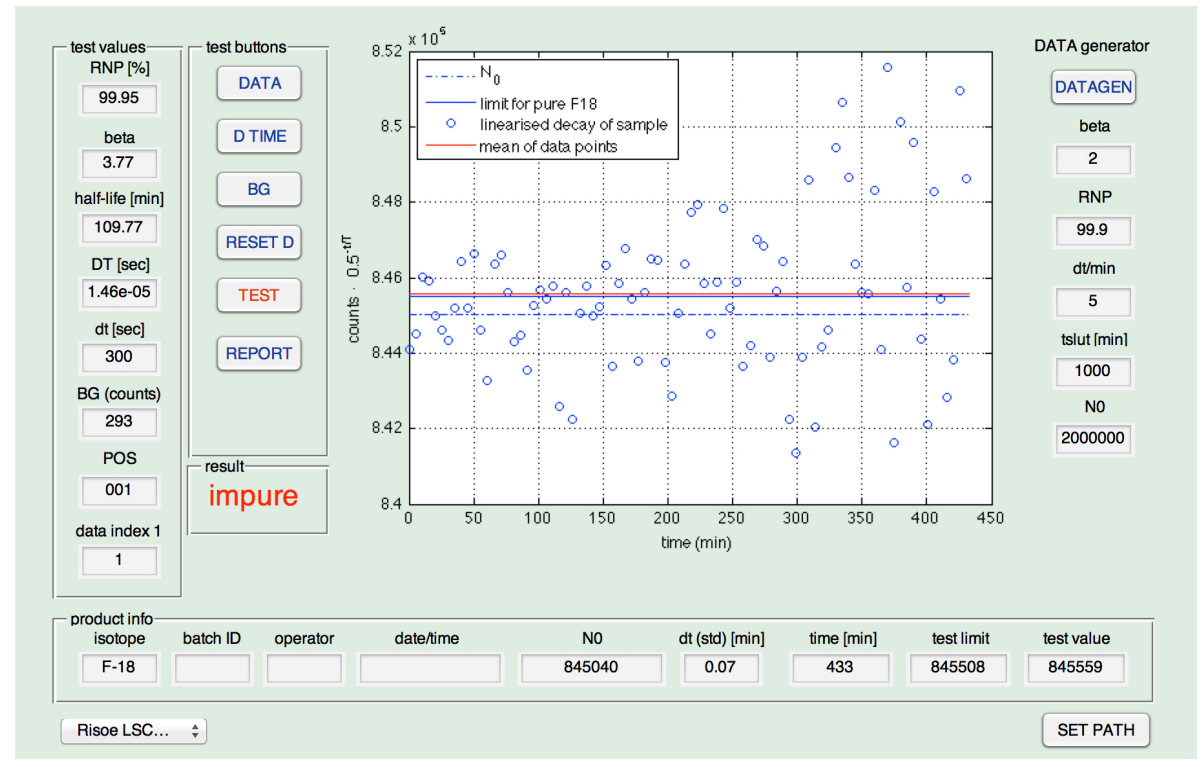

Figure 8: The GUI of the current version of the test application in MATLAB.

tions concerning timing errors, statistical counting errors and total measurement time have been optimized for the case of F-18.

We first implemented the method based on a standard $\mathrm{NaI}$ scintillation counter (COBRA) with a sample changer. Despite much effort in optimizing the timing protocol and adjusting start activity, the COBRA counter invariantly suffered from severe counting errors, because:

1. The sample had to be mechanically relocated before each measurement, leading to slight variations in geometry and significant variations in timing schedule.

2. The COBRA software includes an unavoidable live time correction, extending the physical measurement period until the requested live time had been achieved. This correction moves the timing reference point for each measurement depending on activity of the sample. In principle this correction can be predicted and calculated, but we never succeeded in getting this correction stable, probably reflecting the fact that the "live time" correction was quite sensitive to both sample activity and the actual spectrum of the sample. In summary the COBRA scheme was abandoned. 


\subsection{Dead time}

Dead time is an issue that has to be dealt with in any counting system, but here it is even more important, since the RNP test is very sensitive to small deviations. To get a reliable result, the dead time should be determined accurate and be constant over time.

The dead time for the LSC is found to be $1.45 \cdot 10^{-5} \pm 0.02 \cdot 10^{-5} \mathrm{sec}$, as long as the count rate is less than $4 \cdot 10^{4} \mathrm{cps}$.

The actual run session of the test application offers the possibility of using slightly different dead times in the analysis. However, offsetting the dead time only $0.2 \cdot 10^{-5}$ sec clearly shows up in the early part of the graph for the normalized counts in a manner, which is clearly discernible from any radionuclidic impurity.

In figure 9 an example of a normalized data set that suffers from the lack of dead time correction can be seen, which gives this "logarithmic" shape in the left part of the blue graph. The upper curvature in the right part of the graph is due to the lack of background correction.

Since the uncertainty of the dead time is much smaller than a noticeable offset in the value, it can be difficult to see the impact of a "wrong" value. We have solved that by implementing a procedure in the test that adjust the dead time within the uncertainty interval to give the best possible flat line to

the first few normalized data points. Hence, any small dead time variations should not influence the test.

\subsection{Background}

The test experiments show that it is important with a correct background correction, since the contamination level often is of the same order as the background.

To illustrate that a data set without background correction can be mistaken for an impure sample, we have measured the decay of a pure FDG sample, where the background corresponds to a contamination level of $0.01 \%$. This is seen in right part of the blue and red graph figure 9, and the impact from background is obvious.

If the data is corrected with the average value of the background measurements, then the normalized data will appear as a pure sample, see the green graph in figure 9, and the test result will be the opposite of the one with no background correction. 


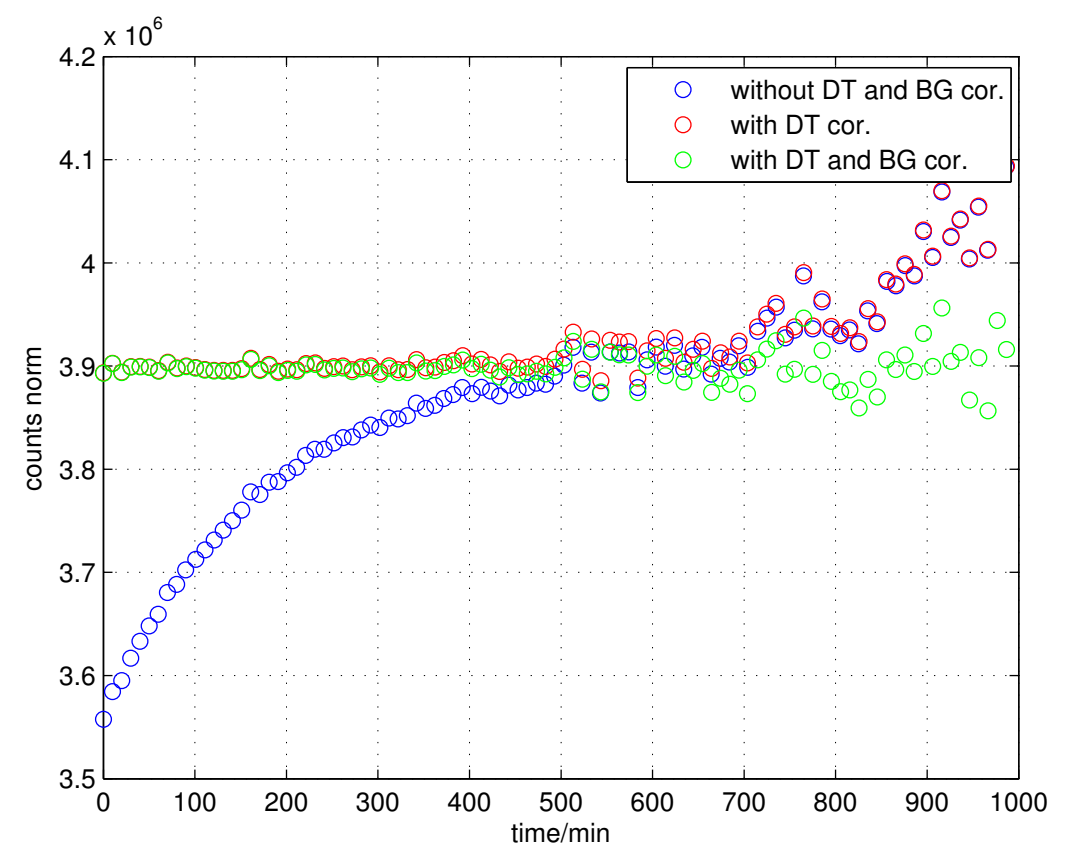

Figure 9: The figure shows what dead time and background (and the corrections) do to the normalized data. The blue graph is normalized data without any corrections. The red graph is normalized data with dead time correction and the green graph represents normalized data with both dead time and background correction. 
Of course, the method cannot detect impurities with count rates corresponding to or less than the statistically uncertainty (Poisson) in the background. For this reason a proper initial count rate should normally be selected at least 1e3 times the background rate (background is sampled for 300-600 secs and with many repeated measurements).

\subsection{Systematic time errors}

The data sets from the LSC contain systematic and periodic time errors. It is apparent that the time deviates 1 min over a period of 15 data points -

or $\pm 0.5 \mathrm{~min}$ around the centre of the period. This is seen on the left side of the blue graph in figure 10 as a sawtooth pattern - before the poisson noise covers the pattern.

This is probably caused by the coincidence between the time the computer uses to write each measurement to its memory and the time resolution of 1 min in the LSC setup. This means that there will be an accumulated error in timing due to rounding of hole minuts over the period of 15 data points.

This pattern will of course have an impact on the determination of $N_{0}$ value, which is the basis of the test, and therefore something needs to be done. We have solved the problem by implementing a code in the test program that automatically recognizes the period and modus in the pattern and corrects the timestamps by:

$$
t_{i, c o r}=t_{i}+n \cdot d t
$$

where the modulo is $n=(-7,-6 \ldots, 6,7)$, and $i$ i is the index number of the data points.

The value of $d t$ is here $1 / 14 \mathrm{~min}$, since the 1 minute is spread over 14 intervals. Hence, an appropriate value for the uncertainty in time would be $\pm 1 / 14 \mathrm{~min}$, which is used in the previous section to calculate the total uncertainty as the test limit. Of course, this value can be chosen as needed.

The red graph in figure 10 shows the correction of the data points, and the formerly so distinct pattern has now disappeared.

\subsection{RNP test results}

For practical reasons we primarily have used $\mathrm{Cu}-64$ to contaminate the FDG compounds to explore the things that influence the tests in practice, but also Ti-45 and Mo-93m were used. The latter isotope and H-3 are more likely produced isotopes, which we have tested with simulated data, see table 1. The test result refers to the time, where the measurement starts, and the 


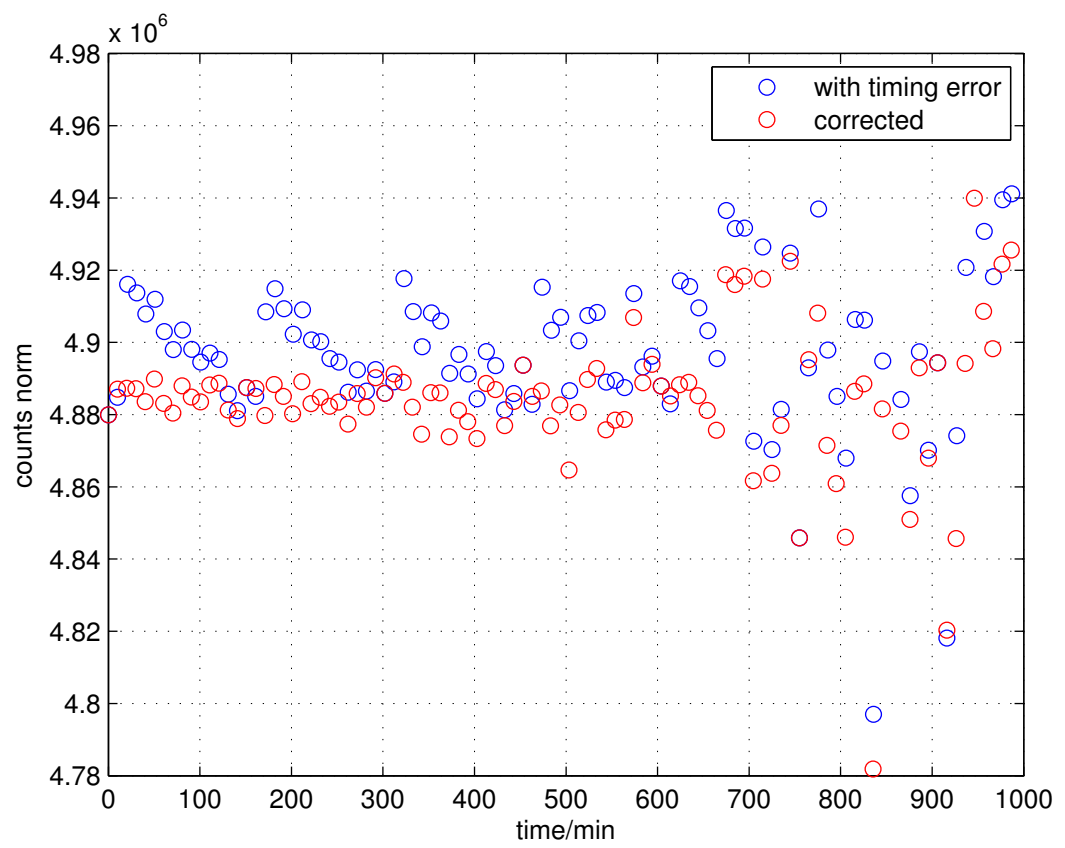

Figure 10: Normalized data set from the LSC. The sawtooth pattern in the blue graph due to the periodic error in timing is obvious in the left side of the graph. In the red graph the timing of the data has been corrected, and now the distinct sawtooth pattern has disappeared. 
contaminated solutions have been made just before the measurements. By adjusting the RNP-parameter in the test we were able to detect all contaminated samples.

From a pure test result we can be certain (statistical confidence of 97.5\%) that there is no impurity present with the chosen test parametres. On the other hand an impure test result tells us that the sample is contaminated, but we cannot use the test to determine the impurity isotope. It only states whether or not the sample is clean on the basis of the test parametres.

The test procedure can be simplified by choosing a value of $\beta$ so small that all important isotopes have larger values. Hence, one will find these kind of contaminations, since the curvature of the normalized graph gets worse with increasing $\beta$. Hence, if the test result comes out impure - the conclusion must be that there is an isotope present with $\beta \geq \beta_{\text {test }}$ and to a degree of $\alpha \leq \alpha_{\text {test }}$.

\section{Discussion}

Any LSC system may suffer from quench, that may be introduced to a variable amount by the sample addition to the cocktail. However, since the quench only influences the detection efficiency in a given counting window independently of the actual count rate, the quench problem does not affect a half-life analysis. Of course an LSC system can never detect an isotope that is shifted completely below the counting window by severe quench, but this is not the case for our very dilute samples of the water based radiopharmaceuticals.

Looking at the test results, it is of great importance that we are able to make an accurate correction of background and dead time, since these two parameters have significant impact of the test result. We have seen how the background can be mistaken for a contamination.

The dead time correction plays an important role, since this regards the first part of the measurement, which is used to establish the value of $N_{0}$ in the test.

The use of a wrong dead time can be recognized from the shape of the graph (in the beginning of the measurement). If the dead time is too small, it gets an logarithmic shape (up) of the first data points, see figure 11, which

cannot be confused with an impurity in the sample, as such will provide a "reverse" curvature. 
On the other hand if it is too large, it gets an exponential shape (down), which can be confused with an impurity that holds a half-life shorter than F18 , but a small deviation corresponds to a very small degree of contamination with a very short half-life, which becomes insignificant in the RNP.

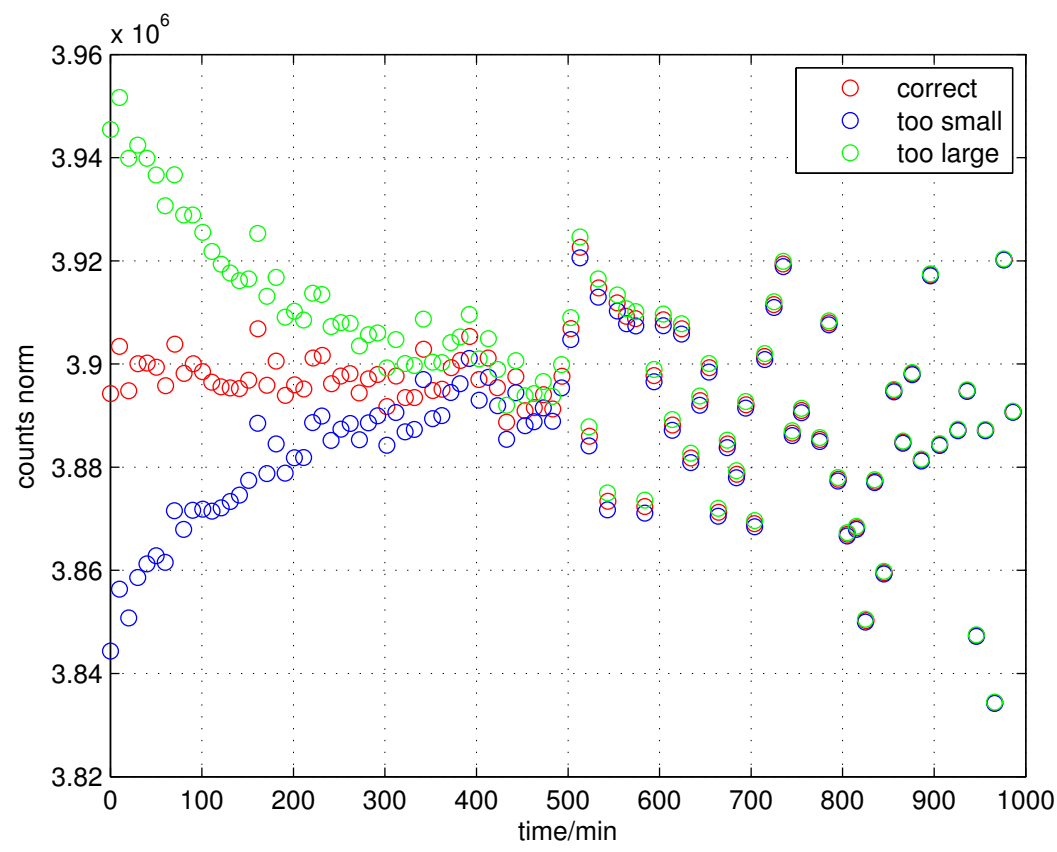

Figure 11: The graph shows, what the use of a wrong dead time does to the graph of normalized counts. A correct chosen dead time (here $1.46 \cdot 10^{-5}$ secs) will give a flat curve at the beginning of the measurement (red graph). If the dead time is too small (here $-0.2 \cdot 10^{-5}$ secs), the first points will have an upward logarithmic shape (blue graph). A too large dead time (here $+0.2 \cdot 10^{-5}$ secs) will on the other hand give an exponential shape (green graph).

However, if the deviation in the dead time is of the order $\pm 0.01 \cdot 10^{-5}$ $\mathrm{sec}$, it is difficult to tell graphically - but it can eg. move the data points upwards to give a wrong test result (impure). Hence, it is of great importance that this value for the counting system is determined and used with great accuracy (to be constant for a given window of count rates).

Since the deviations in the dead time seem to be so small, that the impacts are very difficult to tell graphically, we have build a procedure into the test program to handle that. It uses the same exponential fit that is used to 
determine $N_{0}$ for the same arguments. By running a loop adjusting the dead time within the uncertainty interval, it is possible to find the dead time that gives the best flat line for the normalized fit of these first few data points. Hence, this problem is addressed carefully.

We have shown that the initial count rate should be high in order to have statistical accuracy on the last data points, but on the other hand if the count rate is to high, the dead time will have a greater impact as well as the time uncertainty contribution. It should be balanced between these things, and we have found that an initial count rate of $1 \mathrm{e} 4 \mathrm{cps}$ is working fine.

LSC is preferred over other detector systems due to the detectability of all radioisotopes.

However, the measurements with the LSC are limited to 99 measurements without moving the sample, which limits the total time of measurements. We have used a counting times of 300 and $600 \mathrm{sec}$, but in order to have enough points in the beginning (to establish $N_{0}$ ) together with enough points total (total period of time) an appropriate value could be $450 \mathrm{sec}$. This gives a total time of measurement $t_{m}=742.5$ min. Hence, with $\mathrm{RNP}=99.9 \%$ and $N_{0} \sim 5 \cdot 10^{6}$ it is possible to find isotopes with $\beta \geq 1.4$.

A real contamination from the production facility could of course be of more than one component, but the impure test result will still be an upward curvature of the normalized graph, which deviates from the pure result and therefore turns out to be impure.

In order to keep the test as simple as possible, we only test for one isotope and one RNP at a time, which in return gives a result with a statistical confidence.

In fact if we choose a lower limit of $\beta$ (eg. $\beta=2, t_{m} \sim 300 \mathrm{~min}$ ), so all possible isotopes produced in the production of $\mathrm{F}-18$ are included, and set $\mathrm{RNP}=99.9 \%$ (according to Eur. Ph.), we can be sure to find any contamination of $0.1 \%$ or more from these isotopes. The reason for that is a larger value of $\beta$ increases the curvature of the normalized graph earlier in the measurement compared to a smaller value of $\beta$ - and so does a larger degree of contamination.

It is possible to enlarge the RNP parametre to $99.99 \%$ in the test, but then more data points are needed and the total time of measurement will be increased.

The production and test procedures should be planned in a way that gives as few corrections to the data set as possible in order to have the best sensitivity in the test - eg. the correction of the time pattern could be avoided 
with a better time resolution in the counting system.

The result of the RNP test concerns only isotopes with half-lives larger than F-18 $(\beta>1)$, since the RNPs for samples containing impurities with smaller half-lives than F-18 quickly increase, and therefore they are not relevant to test for. They can be recognized graphically by the 'exponential' curvature in the beginning of the normalized graph.

The test indicates whether or not the sample is pure at the beginning of the measurement and is typically carried out at the time of release, but according to the European Pharmacopoeia a RNP level at 99.9\% (for F-18 FDG) must be satisfied throughout the period of use. This of course increases the demands for the purity at the production time.

The disadvantage is that it takes some time (for $\beta \sim 2$ the $\mathrm{RNP}=99.9 \%$ level can be assured in less than 5 hours with $N_{0} \sim 5 \cdot 10^{6}$ and $\Delta t=5$ min - if the background varies day to day additional 5 hours background counting time is mandatory) to get a test result that meets the requirements (Eur. Ph.) for the test level. However, this test does comply with the requirements - while other methods like direct gamma spectroscopy will not in general detect impurities with $\alpha=0.1 \%$, if for example the main $\gamma$ peak of the impurity is close to or below $511 \mathrm{keV}$. We see the use of non-energy dispersive measurements as the simplest choice. Even with access to high quality Germanium gamma spectrometers small photo peaks below $511 \mathrm{keV}$ may easily remain unnoticed in the Compton continuum. We have tested this by analyzing a mixture of F-18 plus $0.3 \%$ Mo- $93 \mathrm{~m}\left(T_{1 / 2}=6.85 \mathrm{~h}\right.$, a plausible impurity from Niobium targets) with a HPGE detector and GENIE2000 software.

The strong Mo-93m peak at $263 \mathrm{keV}$ remains undetected by the software, and it is only the strong $685 \mathrm{keV}$ line that is detected. Other isotopes only having gammas below $511 \mathrm{keV}$ will be very difficult to detect by gamma spectroscopy.

With an improved and optimized counting system for this method, the test could potentially be done within only $2.5 \mathrm{~h}-3 \mathrm{~h}$ ! Hence, it could be used as an everyday routine quality control of FDG rather than a procedure control.

\section{Conclusion}

In this paper we have identified possible issues in the experimental implementation and their impacts on the sensitivity of the test, and we have used this information successfully to develop a MATLAB application that 
is capable of testing the radionuclidic purity of F-18 compounds very easily. The software can also be used to determine the RNP on other isotopes by changing the half-life as needed.

We conclude that this application for the RNP test seems to work with real data and complies with the requirements in the Eur. Ph. 


\section{Appendix A.}

Uncertainty in time converted to an equivalent uncertainty in the count value by:

$$
\sigma_{\text {equiv,time }}=\left|\frac{d N(t)}{d t}\right| \cdot \sigma_{\text {time }}
$$

which gives:

$$
\sigma_{\text {equiv,time }}=\frac{N_{0} \cdot \log (2)}{(1+\alpha) \cdot T_{R}}\left(\left(\frac{1}{2}\right)^{t / T_{R}}+\frac{\alpha}{\beta} \cdot\left(\frac{1}{2}\right)^{t /\left(\beta T_{R}\right)}\right) \cdot \sigma_{\text {time }}
$$

for a pure decay $(\alpha=0)$ this simplifies to:

$$
\sigma_{\text {equiv }, \text { time }}=\frac{\log (2)}{T_{R}} \cdot N_{0} \cdot\left(\frac{1}{2}\right)^{t / T_{R}} \cdot \sigma_{\text {time }}
$$

\section{Appendix B.}

The uncertainty of $N_{0}$ propagates through the calculations and contributes to the uncertainty of each data point. This is calculated by:

$$
\sigma_{N(t)}=\left|\frac{d N(t)}{d N_{0}}\right| \cdot \sigma_{N_{0}}
$$

For an impure sample this gives:

$$
\sigma_{N(t)}=\frac{1}{1+\alpha}\left(\left(\frac{1}{2}\right)^{t / T_{R}}+\alpha\left(\frac{1}{2}\right)^{t /\left(\beta T_{R}\right)}\right) \cdot \sigma_{N_{0}}
$$

and for a pure sample $(\alpha=0)$ this is reduced to:

$$
\sigma_{N(t)}=\left|\frac{d N(t)}{d N_{0}}\right| \cdot \sigma_{N_{0}}=\left(\frac{1}{2}\right)^{t / T_{R}} \cdot \sigma_{N_{0}}
$$

Hence, the total uncertainty for each data point in the "theoretical decay" is found by:

$$
\sigma_{\text {total }}=\sqrt{\sigma_{\text {equiv,time }}^{2}+\sigma_{N(t)}^{2}+\sigma_{\text {poisson }}^{2}}
$$




\section{Appendix C.}

The total relative uncertainty of the normalized data points is:

$$
\begin{gathered}
\frac{\sigma_{\text {norm }}}{N_{0}}=\sqrt{\left(\frac{\sigma_{\text {total }}}{N(t)}\right)^{2}+\left(\frac{\delta c}{c}\right)^{2}} \\
\delta c=\left|\frac{d c}{d t}\right| \cdot \sigma_{\text {time }}=\frac{\log \left(\frac{1}{2}\right)}{T_{R}} \cdot\left(\frac{1}{2}\right)^{-t / T_{R}} \cdot \sigma_{\text {time }} \\
\frac{\delta c}{c}=\frac{\log \left(\frac{1}{2}\right)}{T_{R}} \cdot \sigma_{\text {time }}=\lambda \cdot \sigma_{\text {time }}
\end{gathered}
$$




\section{References}

[1] Jørgensen, T., et al., 2012, Improved Methods to Determine Radionuclidic Purity of F-18 Compounds, Journal of Applied Radiation and Isotopes, 70 (2012) 430437.

[2] F. Knoll, G., 1979, Radiation Detection and Measurements, first edition, John Wiley \& Sons, New York, pp. 95-102. 